\title{
Drop Impact onto a Thin Viscous Film: Corona Thickness Measurements
}

\author{
Bastian Stumpf*1, Ilia V. Roisman ${ }^{1}$, Cameron Tropea ${ }^{1}$, Alexander L. Yarin ${ }^{2}$, Jeanette Hussong ${ }^{1}$ \\ ${ }^{1}$ Department of Mechanical Engineering, Institute for Fluid Mechanics and Aerodynamics, \\ Technical University of Darmstadt, Darmstadt, Germany \\ ${ }^{2}$ Department of Mechanical and Industrial Engineering, the University of Illinois at Chicago, \\ Chicago, USA \\ *Corresponding author email: stumpf@sla.tu-darmstadt.de
}

\begin{abstract}
Splashing as a result of drop impact onto a thin liquid film is relevant for many industrial applications including spray cooling, spray painting, agricultural sprays or internal combustion engines. During drop impact onto a liquid film the splash is often accompanied by the development of a corona, arising due to the existence of a kinematic discontinuity in the wall film [1]. The corona subsequently breaks up, either as a result of the rim transverse instability, detachment from the wall and fragmentation, or due to the Rayleigh-Taylor instability, resulting in prompt splash.

In this study, rupture of the uprising corona liquid sheet has been artificially induced by perforating it using a thin needle. The analysis of the resulting hole expansion in the corona sheet allows estimation of the sheet thickness using the Taylor-Culick velocity of the rim bounding the expanding hole. The measurement results indicate that the time evolution of the corona sheet thickness depends significantly on the initial wall film thickness onto which the drop impacted, but in all cases it is significantly thinner than the initial liquid film on the wall. A comparison of experimental results and existing theories suggest a possible explanation for this somewhat surprising result.
\end{abstract}

\section{Keywords}

drop impact, splashing, corona, liquid sheet

\section{Introduction}

The splash arising from the impact of a single drop onto a thin liquid film has been investigated rather comprehensively during recent years. Reviews on the hydrodynamic phenomena involved and on the modeling of these phenomena can be found in [2-5]. In such a drop impact the outcome is often a corona splash [6] or prompt splash [7]. Here splash is understood to refer to the existence of smaller, secondary drops resulting from the impact event.

The uprising corona develops as a result of the kinematic discontinuity arising in the wall film [1]. The splashing threshold for corona splash [8] is usually described by the dimensionless number $K=\mathrm{We}^{1 / 2} \mathrm{Re}^{1 / 4}$, where $\mathrm{Re}=\rho U_{0} D_{0} / \eta$ is the Reynolds number and We $=\rho D_{0} U_{0}^{2} / \sigma$ the Weber number $\left(U_{0}\right.$ being the impact velocity, $D_{0}$ the impacting drop diameter, $\rho, \sigma, \eta$, the liquid density, surface tension and dynamic viscosity respectively). Modifications to this number can also characterise the corona splash, even if the liquids of the impacting drop and of the wall film are of different viscosity [9].

In addition to the prompt splash and corona splash a further mode of splash, namely corona detachment, can be observed during drop impact onto thin films [7, 10-12]. In [11] for instance, it is assumed that prompt splash is simply a limiting case of corona detachment, where the detachment occurs so early that no corona can be detected. However, if an uprising corona develops, the formation of secondary droplets can be due to breakup of finger like jets emerging from the unstable free rim. Alternatively, if the uprising corona detaches from the underlying film, a second rim on the lower end of the corona sheet develops, which propagates upwards towards the upper corona rim. When the two merge, secondary drops are also formed. Such a corona detachment can be seen in Fig. 2 presented in the next section. 


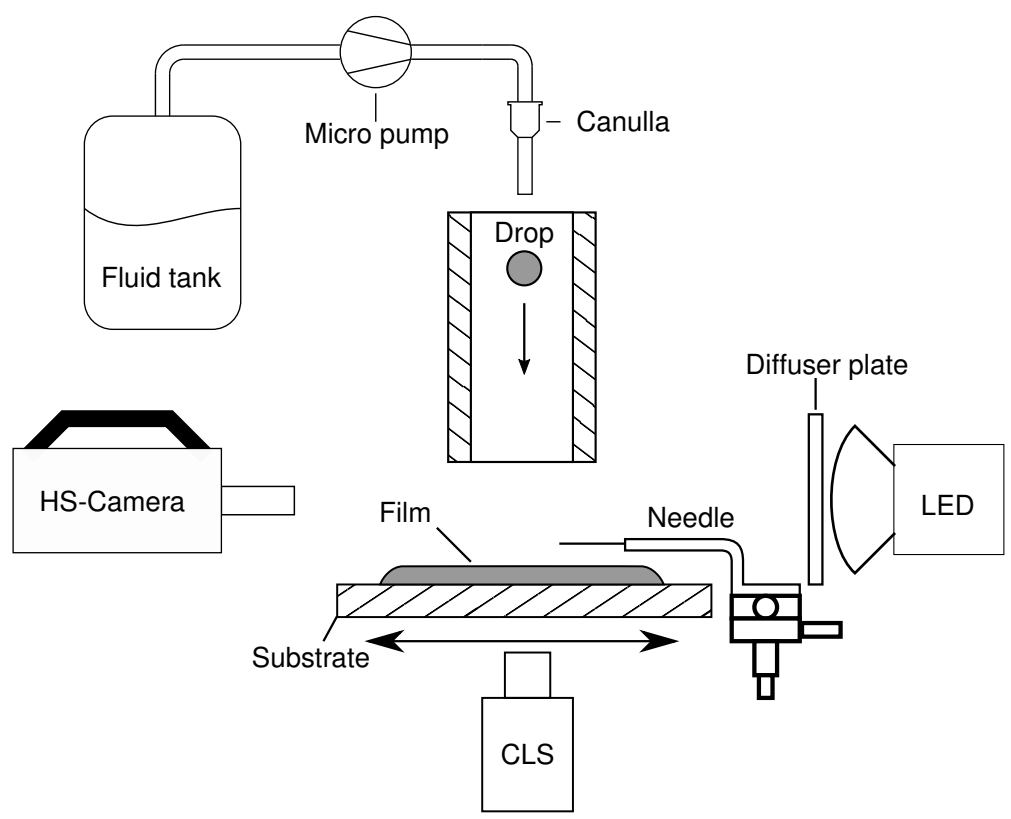

Figure 1. Schematic representation of the experimental setup

It has been observed that in this latter case associated with corona detachment, the number, size and velocity distribution of the resulting secondary droplets can be significantly different than in the first case [12]. Corona detachment can also cause splashing under impact conditions in which no corona splash is expected, leading to deviations from the predictions of the splashing threshold set by the $K$ number.

To better understand the hydrodynamics of corona detachment and the associated development of secondary droplets, one influencing quantity is the thickness of the uprising corona liquid sheet. This is an elusive quantity to capture experimentally; hence, only limited data is available, primarily coming from theoretical predictions or numerical computations. The present experimental study addresses this measurement challenge and introduces a method to determine the corona liquid sheet thickness as a function of time and position throughout the corona sheet. This is accomplished by artificially inducing a rupture into the liquid sheet and observing the propagation of the resulting hole. These measurement results are then compared to existing theoretical expressions for the corona sheet thickness.

\section{Experimental Method \\ Experimental Setup}

To investigate the phenomenon of corona detachment an experiment has been designed, as schematically represented in Fig. 1. The setup consists of a drop-on-demand system, the impact substrate, a high-speed video system and a confocal chromatic line sensor (CLS) used for high-speed measurements of the thickness of the thin liquid film on the substrate.

For drop generation a micropump transports liquid to a canulla where it forms a drop. When the mass of the drop reaches a critical value, the drop pinches off and falls, accelerated by gravity. The impact velocity $U_{0}$ and the drop diameter $D_{0}$ are measured from the high-speed video images.

An optically polished sapphire plate with the dimensions $70 \times 70 \mathrm{~mm}^{2}$ serves as an impact substrate. Due to its high refractive index, sapphire is particularly well suited for measurement of the wall film thickness using the chromatic confocal method from below. To generate wall films of a defined thickness, liquid is first applied to the sapphire glass plate, which spreads over the surface. By constantly monitoring the film thickness with the CLS sensor, a uniform film is ensured. The CLS sensor (Precitec CHRocodile CLS), in combination with a "CLS 0.5 LL"- 
Table 1. Liquid properties of the fluids used in the experiments. The designation "Sxx" denotes different silicone oils with the respective viscosity.

\begin{tabular}{c|cc}
\hline Liquid & $\begin{array}{c}\text { Kinematic viscosity } \\
\nu\left[\mathrm{mm}^{2} / \mathrm{s}\right]\end{array}$ & $\begin{array}{c}\text { Surface tension } \\
\sigma[\mathrm{mN} / \mathrm{m}]\end{array}$ \\
\hline S5 & 5 & 17.72 \\
S10 & 10 & 18.29 \\
S20 & 20 & 18.2
\end{tabular}

\begin{tabular}{|c|c|c|c|c|}
\hline$t=$ & $\begin{array}{c}\text { S5-S5 } \\
H_{f}=42 \mu \mathrm{m}\end{array}$ & $\begin{array}{c}\text { S5-S5 } \\
H_{f}=50 \mu \mathrm{m}\end{array}$ & $\begin{array}{c}\text { S5-S5 } \\
H_{f}=65 \mu \mathrm{m}\end{array}$ & $\begin{array}{c}\text { S20-S20 } \\
H_{f}=50 \mu \mathrm{m}\end{array}$ \\
\hline $0.83 \mathrm{~ms}$ & בnn & & & \\
\hline $1.36 \mathrm{~ms}$ & & & & \\
\hline $1.66 \mathrm{~ms}$ & sionsine & & & \\
\hline $3.28 \mathrm{~ms}$ & & & & \\
\hline $3.83 \mathrm{~ms}$ & & & & \\
\hline
\end{tabular}

Figure 2. Temporal progression of corona expansion and detachment after impact onto wall films of varying initial film thickness and liquid properties. The instant shortly after detachment is highlighted by a red box. The impact parameters are: $U_{0}=3.2 \mathrm{~m} / \mathrm{s}, D_{0}=2 \mathrm{~mm}$.

Probe, is able to measure film thickness at 192 points on a line of $4.5 \mathrm{~mm}$ length. Various initial thickness values of the liquid film are obtained by a spin coating process.

The imaging system comprises a Photron(SA-X2) high-speed camera and a high intensity LED (Veritas, Constellation 120E) which, in combination with a diffusor plate, provides uniform illumination.

Two sets of experiments have been conducted, one to capture the highly dynamic phenomenon of detachment and to precisely determine the time of detachment. For this a frame rate of $300000 \mathrm{fps}$ and a resulting resolution of $256 \times 80$ is used. For a second series of experiments a needle attached to a three-axes positioning system is placed near the drop impact position. During drop impact the ejected corona sheet expands radially and is thereby pierced by the needle. This ruptures the corona sheet, forming a hole which then propagates throughout the corona sheet. Here the impact is captured with a frame rate of $50000 \mathrm{fps}$ and a resolution of $768 \times 328$ pixels.

The liquid properties used in this study are listed in Table 1.

\section{Observations of the corona expansion}

Figure 2 shows the evolution of the corona generated by drop impact onto liquid films of different thickness, where in all cases the drop and wall film liquids were the same. The corona detachment is observed at some time instant, measured from the time of first contact of the drop with the film. The instant of detachment in each case is highlighted with a red box. However, the corona detachment occurs at different stages of corona evolution. At initial wall film heights of $H_{\mathrm{f}}=43$ and $50 \mu \mathrm{m}$ the corona detaches while the rim is still rising; at a film height of $65 \mu \mathrm{m}$ the rim is already falling back towards the substrate. The first three cases exhibit a superposition of corona detachment and a corona splash, which has already begun at the instant of detachment. In the fourth case (S20, $\left.H_{\mathrm{f}}=50 \mu \mathrm{m}\right)$, no rim instability occurs and the formation of secondary droplets (splash) is caused solely by the corona detachment.

It can be seen from these results that the instant of detachment is delayed with growing film 


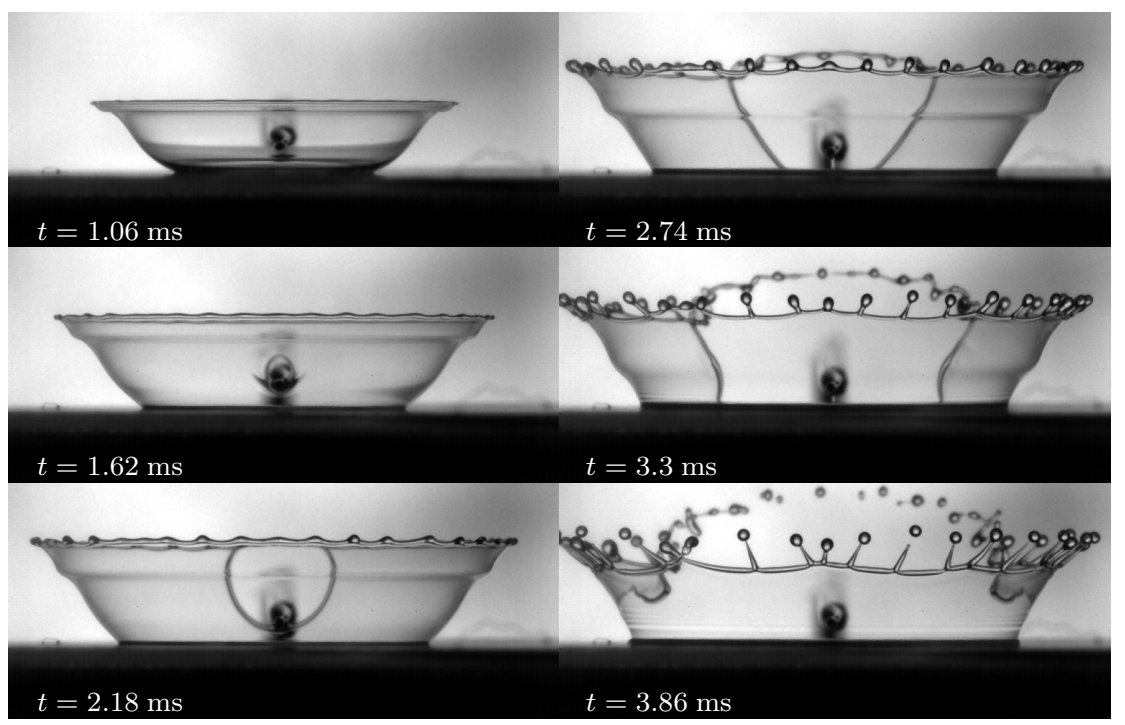

Figure 3. Puncturing of the corona sheet using a needle and propagation of the resulting hole throughout the sheet. The $\Delta t$ between the images is $0.56 \mathrm{~ms}$. Drop impact parameters are: liquid S10-S10, $U_{0}=3.2 \mathrm{~m} / \mathrm{s}, D_{0}=2 \mathrm{~mm}$, $H_{\mathrm{f}}=77 \mu \mathrm{m}$

thickness while the viscosity has no visible influence. The detachment itself happens on a very short timescale, and especially in the first $\left(\mathrm{S} 5, H_{\mathrm{f}}=42 \mu \mathrm{m}\right)$ and the fourth $\left(\mathrm{S} 20, H_{\mathrm{f}}=\right.$ $50 \mu \mathrm{m})$ case the corona detaches almost instantaneously in a horizontal straight line in less than $16 \mu \mathrm{s}$. In the second $\left(\mathrm{S} 5, H_{\mathrm{f}}=50 \mu \mathrm{m}\right)$ and third case $\left(\mathrm{S} 5, H_{\mathrm{f}}=64 \mu \mathrm{m}\right)$ the rupture starts at one point and then propagates in circumferential direction. Since the time of detachment is strongly correlated to the initial film thickness, this can be explained by the fact that very thin films on the substrate, or films of high viscosity are more robust against inclination of the experimental facility and that the asymmetric detachment can be caused by minute gradients of thickness in the wall film.

\section{Measurement Results}

\section{Measurement of the corona sheet thickness}

The method introduced to measure the corona sheet thickness is based on the measurement of the velocity of an expanding hole in the liquid sheet, artificially generated by a needle puncturing the liquid sheet. A visualization of the corona liquid sheet perforated by the needle and resulting hole propagation is shown in Fig. 3.

The hole expansion in an inviscid, plane liquid sheet is governed by the dynamics of the rim, formed by capillary forces. Capillary forces and inertia are in equilibrium, leading to a steady rim propagation velocity $u_{\mathrm{TC}}$, which is determined by the liquid sheet thickness $h$ according to

$$
h=\frac{2 \sigma}{\rho u_{\mathrm{TC}}^{2}},
$$

obtained in $[13,14]$. Eq. (1) is known as the Taylor-Culick relation. Thus, by measuring the rim propagation velocity relative to a liquid sheet, the liquid sheet thickness can be estimated.

It can be seen in Fig. 3 that after the needle punctures the corona liquid sheet at $t=1.63 \mathrm{~ms}$ the hole first grows in a circular manner $(t=2.18 \mathrm{~ms})$. At $t=2.74 \mathrm{~ms}$ the hole rim has reached the corona base as well as the upper rim and propagates in circumferential direction. From $t=2.74 \mathrm{~ms}$ to $3.86 \mathrm{~ms}$ an accelerated propagation of the rim can be observed. To avoid any influence of the puncturing process, the expanding rim velocity is only considered once the rim is at a sufficient distance from the needle.

An in-house image processing algorithm has been developed for the identification of the rim position, the corona center as well as the corona shape at every time step. In Fig. 4 a picture of the drop impact with an induced hole is given for $t=2.6 \mathrm{~ms}$. The coloured lines show the rim 


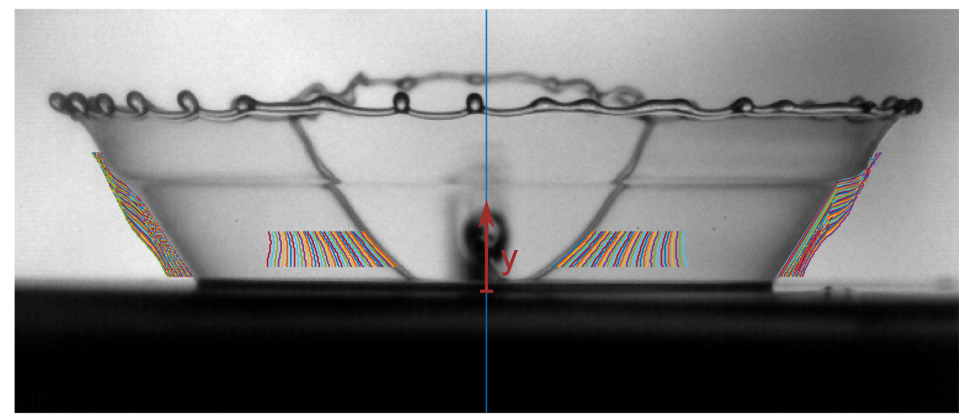

Figure 4. Time evolution of propagating hole rims in the corona sheet $(t=2.6 \mathrm{~ms})$. The blue line denotes the central axis of the corona (impact position). The coloured lines to the left and right of the expanding rim show the rim position and shape at subsequent video frames. Impact parameters: Film and drop liquid is $\mathrm{S} 10, U_{0}=3.2 \mathrm{~m} / \mathrm{s}, D_{0}=$ $2 \mathrm{~mm}, H_{\mathrm{f}}=77 \mu \mathrm{m}$.

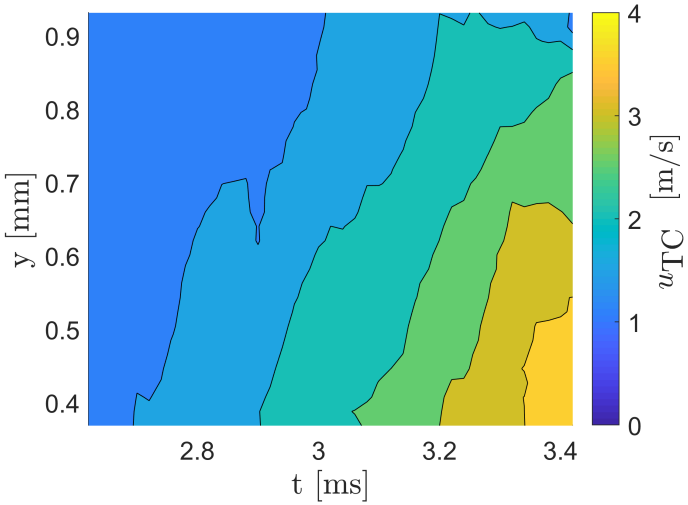

Figure 5. Measured rim velocity on the right side of the corona. The distance $y$ to the substrate is plotted on the ordinate. Impact parameters: Film and drop liquid is $\mathrm{S} 10, U_{0}=3.2 \mathrm{~m} / \mathrm{s}, D_{0}=2 \mathrm{~mm}, H_{\mathrm{f}}=77, \mu \mathrm{m}$.

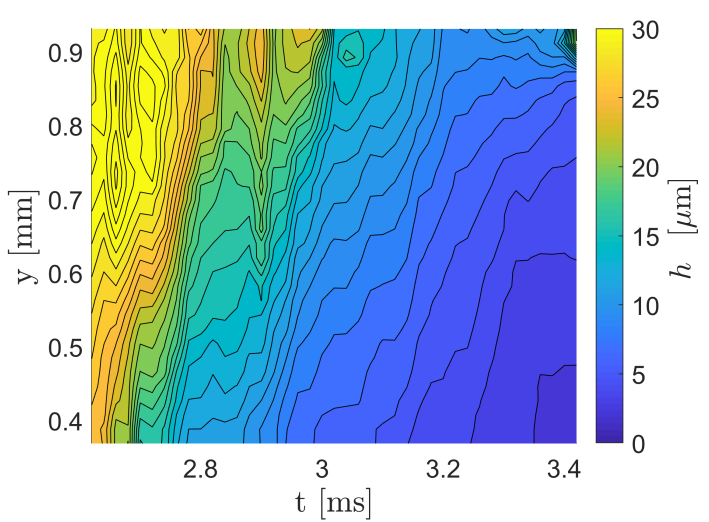

Figure 6. Film thickness on the right side of the corona calculated from Equation (1). Impact parameters: Film and drop liquid is $\mathrm{S} 10, U_{0}=$ $3.2 \mathrm{~m} / \mathrm{s}, D_{0}=2 \mathrm{~mm}, H_{\mathrm{f}}=77 \mu \mathrm{m}$.

position and shape for subsequent time steps. The distance $x$ of the rim to the axisymmetric center of the corona and the corona radius $r$ can be measured directly from the high-speed recordings as a function of time $t$ and height above the corona base $y$. Here $x$ and $y$ refer to the coordinates on the photographic plane. However, since the corona is not a planar sheet but a circular ring, the horizontal displacement of the rim projected onto the camera sensor plane has to be transformed onto the circular corona shape. The angle on the circular corona which the rim propagates between two subsequent time steps $t_{\mathrm{n}}$ and $t_{\mathrm{n}+1}$ can be described as:

$$
\Delta \alpha=\left|\arcsin \left[\frac{x\left(t_{1}, y\right)}{r\left(t_{1}, y\right)}\right]-\arcsin \left[\frac{x\left(t_{2}, y\right)}{r\left(t_{2}, y\right)}\right]\right| .
$$

The change of the radius between two successive time steps is comparably small; hence, a mean radius $\bar{r}=\left[r\left(t_{\mathrm{n}}, y\right)+r\left(t_{\mathrm{n}+1}, y\right)\right] / 2$ can be used to calculate the length of the line element $\Delta s_{m}$ the rim propagates in one time step $\Delta t=t_{\mathrm{n}+1}-t_{\mathrm{n}}$. The line element $\Delta s_{m}$ and the velocity in circumferential direction $u_{m}$ can then be calculated as:

$$
\Delta s_{m}=\bar{r} \Delta \alpha, \quad u_{m}=\frac{\Delta s_{m}}{\Delta t} .
$$

The film thickness in expression (1) is determined only by the velocity component $u_{\mathrm{TC}}$ normal to the rim, which can be experimentally estimated by projecting the measured circumferential velocity $u_{m}$ to a rim-normal direction with the local angle $\beta$, of the rim to the vertical.

$$
u_{\mathrm{TC}}=u_{m} \cos \beta \text {. }
$$

In Fig. 5 the data for the rim velocity is shown as a function of time and the distance $y$ above the corona base for an exemplary set of the impact parameters. It becomes apparent that the rim 


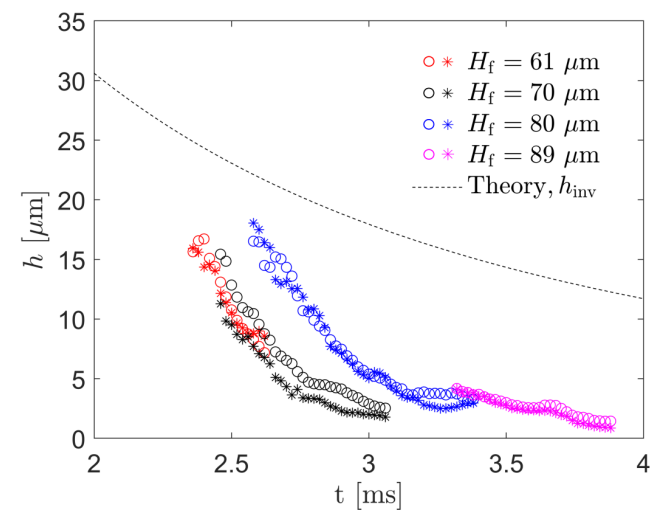

Figure 7. Evolution of film thickness in the corona sheet $0.46 \mathrm{~mm}$ above corona base for different initial film heights $H_{\mathrm{f}}$. The markers $\mathrm{o}$ and $*$ denote the left and the right side rim respectively. The impact parameters are: $U_{0}=3.2 \mathrm{~m} / \mathrm{s}, D_{0}=2 \mathrm{~mm}$, film and drop liquid is $\mathrm{S} 10$.

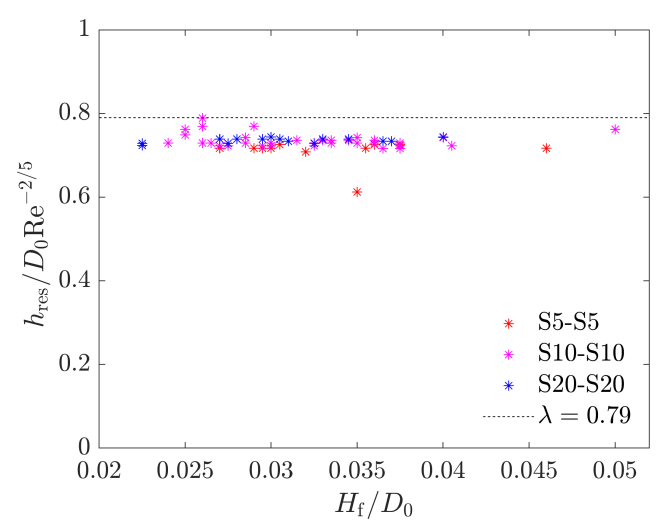

Figure 8. Residual film thickness formed after drop impact for varying $H_{\mathrm{f}}$ and viscosity.

accelerates over time and propagates faster the closer it is to the corona base. These data are consistent with the observations shown in Fig. 3 where from $t=2.74 \mathrm{~ms}$ on, the lower part of the rim passes the upper part.

The corresponding data for the corona thickness, estimated using Eq. (1) and the data for the rim velocity from Fig. 5 , is shown in Fig. 6 . It can be seen that the corona rapidly thins out in time with the lowest values of film thickness in the vicinity of the corona base. The significant corona thinning at large times can explain the eventual corona breakup and detachment from the wall, shown in Fig. 2.

\section{Evolution of the corona thickness}

With regard to the fact that the instant of detachment is delayed with increasing initial wall film thickness $H_{\mathrm{f}}$, the influence of $H_{\mathrm{f}}$ on the film thickness of the corona sheet $h$ is of particular interest. The temporal evolution of $h$ on a horizontal line $0.46 \mathrm{~mm}$ above the corona base is shown for cases with varying initial film thickness in Fig. 7 . The measured thickness for the corona sheet on the left and the right hand side of the corona axis coincide remarkably well, demonstrating the high symmetry of the impact and the well adjusted experimental parameters. Four characteristic film heights are chosen for comparison in Fig. 7. While at $H_{\mathrm{f}}=61 \mu \mathrm{m}$ the corona detaches at $t=2.5 \mathrm{~ms}$ and at $H_{\mathrm{f}}=70 \mu \mathrm{m}$, the detachment occurs at $t_{0}=3.1 \mathrm{~ms}$, for $H_{\mathrm{f}}=80 \mu \mathrm{m}$ and $H_{\mathrm{f}}=89 \mu \mathrm{m}$ no detachment can be observed anymore. Keeping in mind that the applied method does not account for additional forces due to acceleration of the rim, the resulting film thickness is slightly overestimated. Nevertheless a rapid thinning of the corona sheet can be observed from approximately $18 \mu \mathrm{m}$ to $2-4 \mu \mathrm{m}$. The rapid decrease of film thickness is delayed with increasing $H_{\mathrm{f}}$. The thickness of the corona is much smaller than the thickness of the initial wall film, which contradicts the predictions based on the inviscid solution $[1,15]$. Moreover, the corona thickness is also much smaller than the lamella thickness generated by normal impact of an inviscid liquid drop [16] onto a planar substrate

$$
h_{\text {inv }}=D_{0} \frac{0.39}{(\tilde{t}+0.25)^{2}} \exp \left[-\frac{2.34 \tilde{r}^{2}}{(\tilde{t}+0.25)^{2}}\right], \quad \tilde{t}=\frac{t U_{0}}{D_{0}}, \quad \tilde{r}=\frac{r}{D_{0}} .
$$

This expression is obtained in using numerous experimental data and direct numerical computations of drop impacts with very high Reynolds number.

\section{Viscosity effects near the wall}

It is well known that viscosity effects influence the flow in the lamella when the thickness of the viscous boundary layer is comparable with the lamella thickness. At larger times after impact 
a)

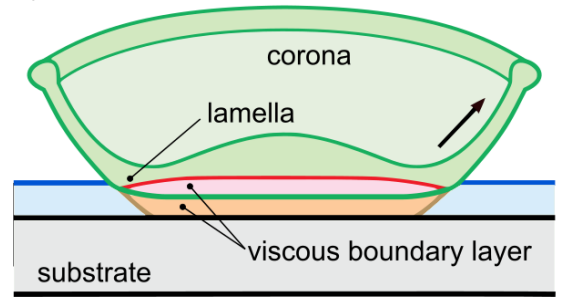

b)

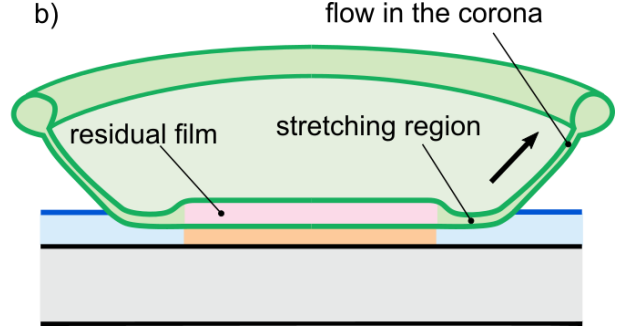

Figure 9. Sketch of the main stages of the corona expansion.

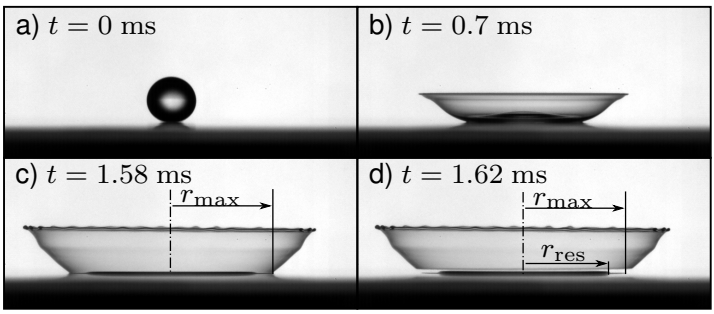

Figure 10. Temporal evolution of corona with the maximum corona radius $r_{\max }$ shown in $\mathrm{c}$ ) and the radius of the residual film $r_{\text {res }}$ shown in d). Impact parameters: Drop and film liquid is $\mathrm{S} 10, U_{0}=$ $3.2 \mathrm{~m} / \mathrm{s}, D_{0}=2 \mathrm{~mm}, H_{\mathrm{f}}=52 \mu \mathrm{m}$.

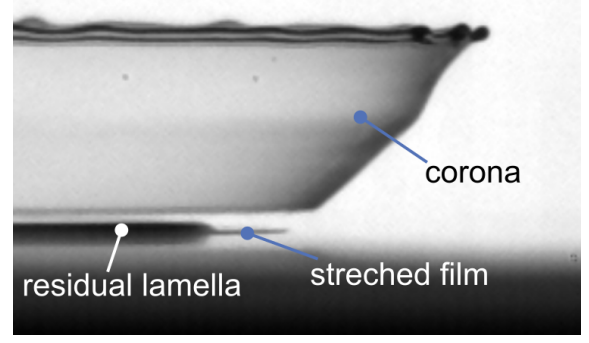

Figure 11. Geometry of the lamella in the vicinity of the corona. Definition of the residual lamella and the stretched liquid film

the viscous effects lead to a fast flow deceleration and formation of the residual film of nearly constant thickness

$$
h_{\text {res }}=\lambda D_{0} \operatorname{Re}^{-2 / 5} \text {. }
$$

In the case of drop impact onto a dry solid substrate the theoretical prediction $\lambda \approx 0.79$ is obtained in [17]. The scaling expressed in Eq. (6) is also applicable to the case of relatively thick liquid films [18] while the value of $\lambda$ is a function of the dimensionless initial wall film thickness $H_{\mathrm{f}} / D_{0}$.

The experimental data for the residual lamella thickness, obtained in this study for a very thin wall film, $H_{\mathrm{f}} / D_{0} \ll 1$, using the CLS sensor, is shown in Fig. 8. In the range of the ratio $0>H_{\mathrm{f}} / D_{0}<0.05$ the measured value of $\lambda$ is nearly constant and very close to the theoretical predictions $\lambda=0.79$ for the case $H_{\mathrm{f}} / D_{0}=0$.

\section{A puzzle of corona expansion for long times}

The typical time for expansion of the viscous boundary layer and the creation of the residual film is estimated in [17] as $t_{\text {res }} \sim 0.6 D_{0} / U_{0} R e^{1 / 5}$. For the present set of impact parameters this time is approximately $t_{\text {res }} \sim 1.2-1.6 \mathrm{~ms}$, depending on the liquid viscosity. But the observed corona continues to expand for times significantly exceeding $t_{\text {res }}$ even when the flow in the lamella disappears.

A possible explanation to this puzzle is shown schematically in Fig. 9. The viscous effects are initially significant only in the thin viscous boundary layers in both wall liquid and drop liquid regions. The layers are initiated only at the base of the crown and do not influence the flow in the corona, Fig. 9(a). At larger times a residual film is formed by viscosity, but the liquid flow in the corona is unperturbed. Therefore, a stretching region of the corona has to appear near the base of the corona, where the velocity gradient and therefore the rate of the thickness change are relatively high.

The appearance of the thin stretching region has been observed in the present experiments. In the case, shown in Fig. 10 the corona detaches from the substrate. This allows observation of the residual film before it starts to spread. It is astonishing that the radius $r_{\text {res }}$ of the residual film is notable smaller than the radius of the corona $r_{\max }$.

A more detailed view on the region of the corona base just after its detachment is given in Fig. 11 . 
The view is taken from Fig. 10d. In this image the stretched liquid film between the corona and the nearly stationary residual film is clearly seen. The region between the residual lamella and the base of the corona corresponds to the emergence and growth of the viscous boundary layer. The flow in the residual lamella is governed by the viscous and inertial terms over its entire thickness.

\section{Conclusions}

This is an experimental study of drop impact onto a very thin liquid film. A method has been developed for the estimation of the thickness of the corona sheet as a function of time and position within the uprising corona liquid sheet. It is interesting that the thickness of the corona is much smaller than the initial wall film thickness and the residual lamella thickness after drop impact. This result is explained by the local lamella stretching in the region of the corona base.

\section{Acknowledgements}

The authors gratefully acknowledge the financial support of this work by the Deutsche Forschungsgemeinschaft (DFG) in the framework of TRR 150 - Project-ID 237267381.

\section{References}

[1] Yarin, A. L., Weiss, D. A., 1995, Journal of Fluid Mechanics, 283 pp. 141-173.

[2] Yarin, A. L., 2006, Annual Review of Fluid Mechanics, 38 pp. 159-192.

[3] Josserand, C., Thoroddsen, S. T., 2016, Annual Review of Fluid Mechanics, 48 pp. 365-391.

[4] Marengo, M., Antonini, C., Roisman, I. V., Tropea, C., 2011, Current Opinion in Colloid \& Interface Science, 16 (4) pp. 292-302.

[5] Yarin, A. L., Roisman, I. V., Tropea, C., 2017, Collision Phenomena in Liquids and Solids, Cambridge University Press, Cambridge.

[6] Cossali, G., Coghe, A., Marengo, M., 1997, Experiments in Fluids, 22 (6) pp. 463-472.

[7] Burzynski, D. A., Roisman, I. V., Bansmer, S. E., 2020, Journal of Fluid Mechanics, 892.

[8] Mundo, C., Sommerfeld, M., Tropea, C., 1995, International Journal of Multiphase Flow, 21 (2) pp. 151-173.

[9] Kittel, H. M., Roisman, I. V., Tropea, C., 2018, Phys. Rev. Fluids, 3 p. 073601.

[10] Roisman, I. V., Gambaryan-Roisman, T., Kyriopoulos, O., Stephan, P., Tropea, C., 2007, Physical Review E, 76 (2) p. 026302.

[11] Kittel, H., 2019, Drop Impact onto a Wall Wetted by a Thin Film of Another Liquid, Dissertation, Technische Universität Darmstadt, Darmstadt.

[12] Geppert, A. K., 2019, Experimental Investigation of Droplet Wall-Film Interaction of Binary Systems, Dissertation, University of Stuttgart, Stuttgart.

[13] Taylor, G. I., 1959, Proceedings of the Royal Society London A, 253 pp. 625-639.

[14] Culick, F., 1960, Journal of Applied Physics, 31 pp. 1128-1129.

[15] Roisman, I. V., Tropea, C., 2002, Journal of Fluid Mechanics, 472 pp. 373-397.

[16] Roisman, I. V., Berberović, E., Tropea, C., 2009, Physics of Fluids, 21 (5) p. 052103.

[17] Roisman, I. V., 2009, Physics of Fluids, 21 (5) p. 052104.

[18] Van Hinsberg, N. P., Budakli, M., Göhler, S., Berberović, E., Roisman, I. V., GambaryanRoisman, T., Tropea, C., Stephan, P., 2010, J. Colloid Interface Sci., 350 (1) pp. 336-343. 